\title{
An Investigation into the Prevalence of Migraine and Its Prophylactic Treatment Patterns in the Czech Republic: An Observational Study
}

This article was published in the following Dove Press journal: Journal of Pain Research

\author{
David Dolezil' \\ Jolana Markova ${ }^{2}$ \\ Jiri Klimes ${ }^{3,4}$ \\ Zuzana Pocikova ${ }^{3,4}$ \\ Filip Dostal $\left.\right|^{3,4}$ \\ Radka Stepanova $a^{5,6}$ \\ Adam Svobodnik (iD ${ }^{5,7}$ \\ 'Prague Headache Center, DADO \\ MEDICAL s.r.o., Prague, Czech Republic; \\ ${ }^{2}$ University Thomayer Hospital, Prague, \\ Czech Republic; ${ }^{3}$ Novartis, s.r.o., Prague, \\ Czech Republic; ${ }^{4}$ Faculty of Pharmacy, \\ Department of Social and Clinical \\ Pharmacy, Charles University in Prague, \\ Prague, Czech Republic; ${ }^{5}$ Aixial, s.r.o., Brno, \\ Czech Republic; ${ }^{6}$ Department of \\ Pharmacology, Faculty of Medicine, \\ Masaryk University, Brno, Czech Republic; \\ ${ }^{7}$ International Clinical Research Center, St. \\ Anne's University Hospital Brno, Brno, \\ Czech Republic
}

Correspondence: Jiri Klimes Novartis, s.r.o, Prague, Czech Republic Tel +420728328703

Email jiri.klimes@novartis.com
Purpose: A national primary and secondary healthcare-level study in the Czech Republic has not yet been conducted to evaluate the prevalence of migraine. We analyzed the current treatment patterns (acute and prophylactic) in migraine patients and the number of migraine patients potentially eligible for treatment with recent calcitonin gene-related peptide (CGRP) pathway-targeted therapies.

Methods: This retrospective study utilized the Ministry of the Interior Health Insurance Fund claims database of the Czech Republic wherein every citizen is insured. Migraine patients with or without aura, and potentially on triptan therapy were included in this study (index years 2012-2016). The prevalence approach included all patients (new and old) present in each index year. Prophylactic therapies were followed foor three and seven years prior to the index year, including the index year, until 2010. The incidence approach included all patients first diagnosed in each index year. Prophylactic therapies were followed for the next three years, including the index year, until 2017 following incidence approach. The primary endpoint of this study was to determine the rate of migraine prevalence and diagnosis for each index year during the period 2012-2016. The study also evaluated prophylactic and acute treatment patterns and comorbidities among patients in 2016.

Results: The rate of migraine prevalence was $1 \%$ and the rate of diagnosis was $0.2-0.4 \%$. By prevalence approach, approximately $39 \%$ of the patients were on prophylactics, and $11.2 \%$ and $21.6 \%$ of the patient population had two prior treatment failures (three- and seven-year recall period, respectively). Antiepileptics (26\%) and beta blockers (15.8\%) were the most prescribed prophylactics, and sumatriptan was the predominant triptan used $(12 \%)$ for acute treatment.

Conclusion: Taking into account the number of inhabitants in the Czech Republic (10.7 million), there could be up to 23,000 adult patients eligible for novel CGRP therapies.

Keywords: CGRP, prophylactics, triptans, claims database

\section{Introduction}

According to the Global Burden of Disease study, neurological disorders are the leading cause of disability-adjusted life years (DALY) and the second leading cause of death. ${ }^{1}$ Stroke (42.2\%), migraine (16.3\%), Alzheimer's and other dementias $(10.4 \%)$, and meningitis $(7.9 \%)$ were the top disorders contributing to the cause of neurological DALYs. ${ }^{1}$ In terms of years lived with disability (YLD), migraine is ranked second globally and has a prevalence of $14.4 \%$, with 1.04 billion living with migraine and 45.1 million YLDs. $^{2}$ Despite being one of 
the most frequent neurological disorders, the burden of this affliction is highly underestimated. ${ }^{3}$

Treatment of chronic and episodic migraine (CM and EM) involves abortive or acute treatment and preventive or prophylactic treatment. ${ }^{4}$ Triptans are the first-line of treatment for acute migraine and although they may be effective in treating acute attacks, frequent use may lead to medication overuse and medication overuse headache. ${ }^{5}$ It is thus recommended that prophylactic therapy be used to limit the frequency of acute therapy. ${ }^{6}$ A number of prophylactic drugs to treat migraine, such as antidepressants, antihypertensives (beta blockers, angiotensin receptor blockers, angiotensin-converting enzyme inhibitors) and antiepileptics were initially developed for other indications and were not intended for treatment of migraine. ${ }^{6,7} \mathrm{In}$ recent years, treatments specific for migraine have been developed, targeting the calcitonin gene-related peptide (CGRP) pathway, which has been known to play a central role in migraine pathophysiology. ${ }^{8}$ One of these therapies is erenumab, a fully human monoclonal antibody that blocks CGRP activity by targeting the CGRP receptor. In clinical trials conducted in patients with EM and CM, erenumab has demonstrated its efficacy by reducing monthly migraine days and was well tolerated with an adverse event profile similar to placebo. ${ }^{9,10}$ Another area of consideration when treating migraine patients is comorbidities. Conditions such as cardiac diseases, psychiatric diseases, obesity and epilepsy are some of the commonly occurring comorbidities in patients already suffering from migraine. ${ }^{11}$ Thus, it is particularly important to contextualize all of the medical conditions of a migraine patient in order to effectively manage the condition.

Over the years, cross-sectional studies such as the International Burden of Migraine Study and the Eurolight project have been conducted across various European countries to collect migraine prevalence data. ${ }^{12,13}$ Studies have also compared the clinical and economic burden of migraine in 31 countries across the world using selfreported data from online bulletin boards. ${ }^{14}$ Considering the results of the global burden of disease study (2018), with burgeoning number of migraine patients across the world, ${ }^{2}$ it is imperative that we study a country's treatment patterns and individual drug usage in a proper healthcare management setting in order to improve healthcare delivery. In the Czech Republic, no real-world national study evaluating the prevalence or diagnosis of migraine and its treatment patterns has been conducted at primary and secondary care levels. A recent study in the country analyzed the burden of migraine using data from a selfreported smartphone application, ${ }^{15}$ but self-reported migraine may or may not have been confirmed by a medical diagnosis and thus, any data obtained at a local or national level are not entirely reliable. Claims data are being increasingly used in medical research as a source of useful information ${ }^{16,17}$ because they record details on diagnosis, treatments provided, care providers, billed and reimbursed amounts, and show concordance with patient self-reports/electronic health records with acceptable accuracy. ${ }^{16,18}$

The present study collected and analyzed real-world claims data from ZP MV (Ministry of the Interior Health Insurance Fund) in the Czech Republic. The objective of this study was to primarily evaluate the prevalence and diagnosis of migraine at primary and secondary care levels, and to understand the present treatment patterns in the country. This study could inform the use of novel CGRP therapies and their upcoming launches with respect to a proper setting of migraine disease management and budgeting. The current article focuses to present the observations from this study.

\section{Methods}

\section{Type of Study and Study Population}

This was an observational, retrospective study utilizing the ZP MV claims database (approximately $12 \%$ of the whole Czech Republic population). It is to be noted that all the citizens of the Czech Republic (10.7 million) ${ }^{19}$ are insured, by law. Three migraine populations were included in this study: (a) pure migraine population with or without aura (Population A), (b) potential migraine population on triptan therapy (Population B), and (c) total migraine population, ie, Population A + Population B (Population C). All primary and secondary endpoints were analyzed separately for the three population subgroups. Data for Population C, which includes the complete migraine population, including those on triptan therapy, are presented in this article.

\section{Study Design}

This study focused on two approaches:

(a) Prevalence approach - included all patients of the study cohort present in each year (already existing and newly diagnosed) within the period 2012-2016. Prophylactic therapies were followed for three and seven years prior to the reference year, including the 
reference year, eg, for the reference year 2016, prophylactic therapies were followed up for 2016 and back to 2014 (three-year recall period), and for 2016 and back to 2010 (seven-year recall period) (Figure 1A).

(b) Incidence approach - included all patients of the study cohort first diagnosed in each year during the period 2012-2016 (newly diagnosed patients, ie, patients not having any migraine claims data before the reference year). Prophylactic therapies were followed up for the subsequent years, including the reference year, eg, for the reference year 2015, prophylactic therapies were followed up for 2015, 2016 and 2017 (Figure 1B). This approach provides a clearer picture of prophylactic treatments in terms of sequences used because the prevalence/crosssectional approach does not capture the patients independently when they initiate their prophylactic treatment. All descriptive analyses were performed within the index/reference year, except for the prophylactics, which were also observed beyond the index/reference year.

In this manuscript, primary endpoint included data for all the years from 2012 to 2016 . Demographic characteristics of the patient population and the data for secondary endpoints were presented for the index year 2016.

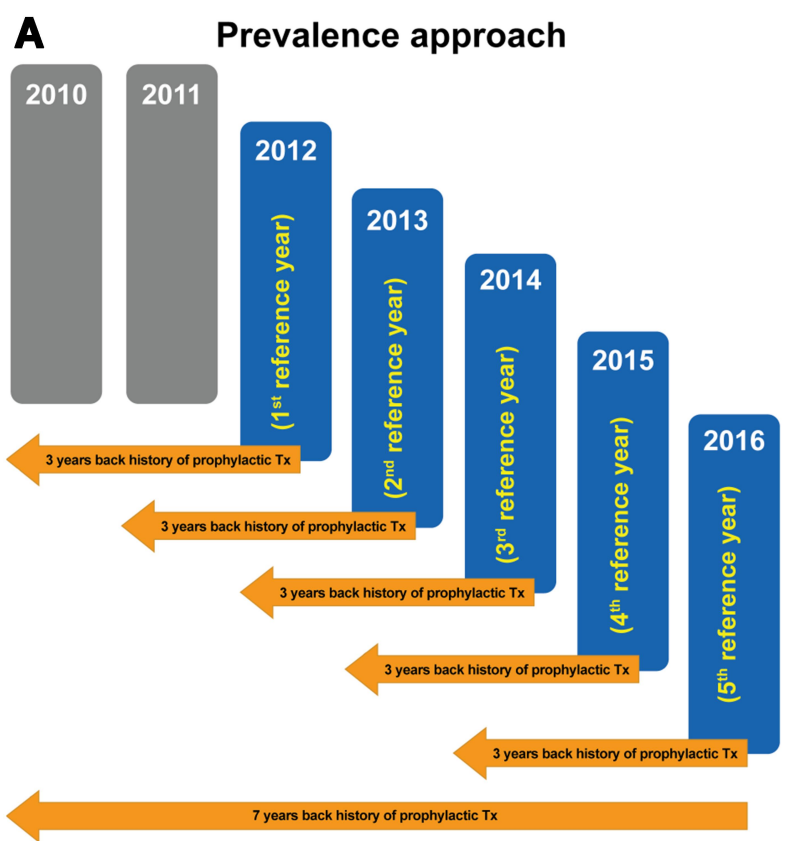

\section{Primary Endpoint}

The primary endpoint was the prevalence and diagnosis rate of migraine for each reference/index year within the time period 2012-2016, using the prevalence and incidence approaches, respectively. According to the prevalent analysis approach, each patient having migraine-related medical procedures and/or prophylactic or acute medication recorded within the time period 2012-2016 was counted into the prevalence of migraine for the corresponding index year. According to the incident analysis approach, each patient having migraine-related medical procedures and/or prophylactic or acute medication recorded within the time period 2012-2016 was counted into the incidence of migraine for the corresponding index year unless any migraine-related medical procedures were present prior to the index year. This rate was evaluated for the corresponding index year based on the type of analysis approach and was presented as the number and percentage of migraine patients out of the total-insured population.

\section{Secondary Endpoints}

1. Prophylactic treatment

(a) Number of migraine patients treated with a particular number of prophylactic medications $(0, \geq 1, \geq 2, \geq 3$, and $\geq 4$; cumulative prophylactic
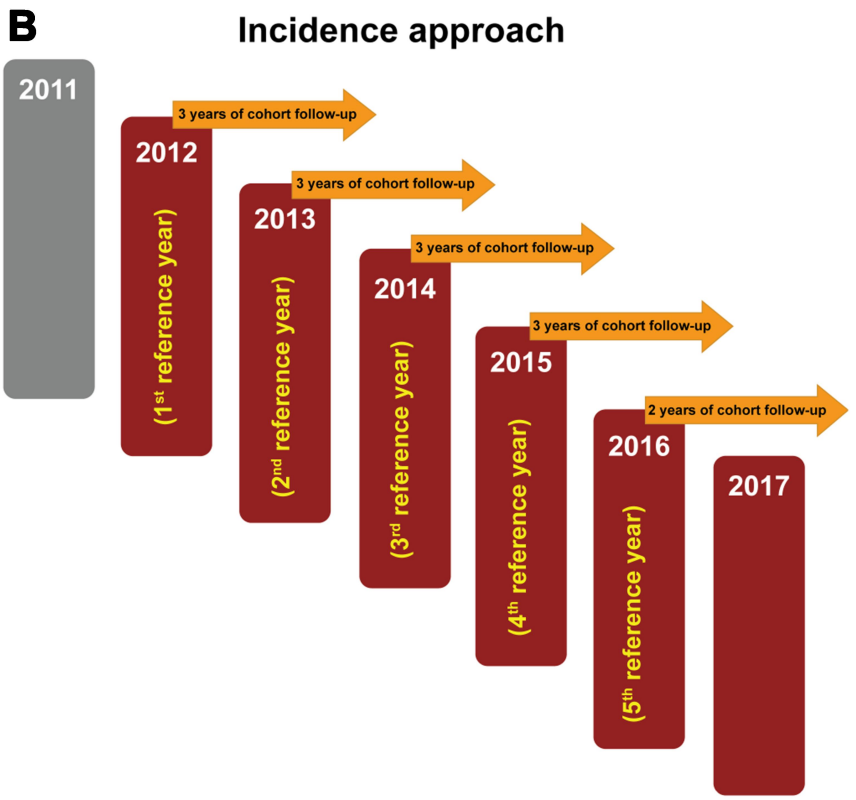

Figure I Study Design for (A) Prevalence and (B) Incidence Approaches. (A) Prevalence approach (index years 2012-2016): Prophylactic therapies were followed up for three and seven years prior to the index year, including the index year, until 2010. (B) Incidence approach (index years 2012-2016): Prophylactic therapies were followed up for the next three years, including the index year, until 2017. For the year 2016, therapies were followed for two years. Abbreviation: Tx, treatments. 
line, ie, 0 prophylactic medications used, 1 st and subsequent prophylactic line, 2nd and subsequent prophylactic line, and so on) in the index year 2016, with three- and seven-year recall periods.

(b) Proportion of each prophylactic medication used in the index year 2016 (both the therapeutic subgroup and individual drugs; no prophylactic lines), with a three-year recall period.

1. Acute treatment - Number of daily doses of triptan and the types of triptan (triptans were the only acute treatment form recorded) with respect to the number of concomitant prophylactic medications used $(0,1 \mathrm{st}, 2 \mathrm{nd}, 3 \mathrm{rd}, 4 \mathrm{th}$; distinctive prophylactic line or exact number of concomitant prophylactic medications used) in the index year 2016.

2. Comorbidities - Number of migraine patients with comorbidities, and the number and types of comorbidities in the index year 2016.

\section{Ethics Approval}

This was a retrospective, anonymized study requiring no approval from the ethics committee or health authorities, or informed consent from patients. No personal data were used in the study. Data used in this study were from a preexisting database.

\section{Statistical Analysis}

The data obtained from the study population were analyzed using descriptive statistics. Categorical data are summarized as an absolute or relative frequency. Continuous data are presented as the number of available observations, means and standard deviations (SD). Percentages have been calculated using $\mathrm{n}$, the number of valid (not missing or unknown) counts for each item. Missing or unknown values have not been imputed.

\section{Results}

\section{Patient Demographics}

Out of the 85,126 patients in the database (2012-2016), 28,285 fulfilled the criteria of a pure or potential migraine population. The remaining patients either did not have data pertaining to all years from 2012 to 2016 or did not fulfill the criteria for the migraine population (Figure 2A). The mean age of migraine patients in the index year 2016 was 42.0 years and 33.7 years using the prevalence and incidence approach, respectively (Figure $2 \mathrm{~B}$ and $\mathrm{C}$ ). The proportion of female migraine patients was $80.8 \%$ (prevalence approach) and 71.5\% (incidence approach). On stratifying the patient population according to minors (aged 0-17 years), adults (aged 18-65 years) and elderly (aged $\geq 66$ years), it was observed that migraine was most prevalent in the adult age group of 18-65 years and least prevalent in the elderly. This was true for both the prevalence $(84 \%$ vs $7 \%)$ and incidence approaches $(77 \%$ vs $3 \%)$.

\section{Primary Endpoint}

The migraine prevalence rate in the Czech Republic remained stable during the period of 2012-2016. Only $1 \%(\mathrm{n}=14,030)$ of patients from the total insured population $(n=1,288,409)$ had claims made towards migraine-related healthcare expenses in 2016. The migraine diagnosis rate among newly diagnosed patients, ie, incidence approach patients, across 2012-2016 was $0.2-0.4 \%$. Hence, approximately onethird of the total migraine population was represented by newly diagnosed patients (Table 1).

\section{Secondary Endpoints}

Prophylactic Treatment

Various antiepileptic and antidepressant drugs were prescribed to the migraine patients. Among the antiepileptic drugs, topiramate was predominantly used as a preventive treatment. Beta blockers were the only form of antihypertensive prescribed. For assessment of prophylactic treatment, cumulative medication lines $(0, \geq 1, \geq 2, \geq 3$ and $\geq 4)$ were considered.

Number of Migraine Patients Treated with a Particular Number of Prophylactic Medications

The number of cumulative prophylactic lines assessed for the index year 2016 was 0 to $\geq 4$. Upon stratifying by age and using the prevalence approach for analysis, the mean age of migraine patients ranged between 48 and 53 years of age (three- and seven-year recall periods) for the $\geq 1$ to $\geq 4$ prophylactic lines (Table $2 \mathrm{~A}$ ). The proportion of patients treated with $\geq 1$ prophylactic medication was $38.8 \%$ (three-year recall period) and $46.8 \%$ (seven-year recall period) (Table 2B). The proportion of patients treated with $\geq 2$ prophylactic medications was $11.2 \%(84.6 \%$ females; three-year recall period) and $19.8 \%(86 \%$ females; seven-year recall period) (Table 2A and B). 


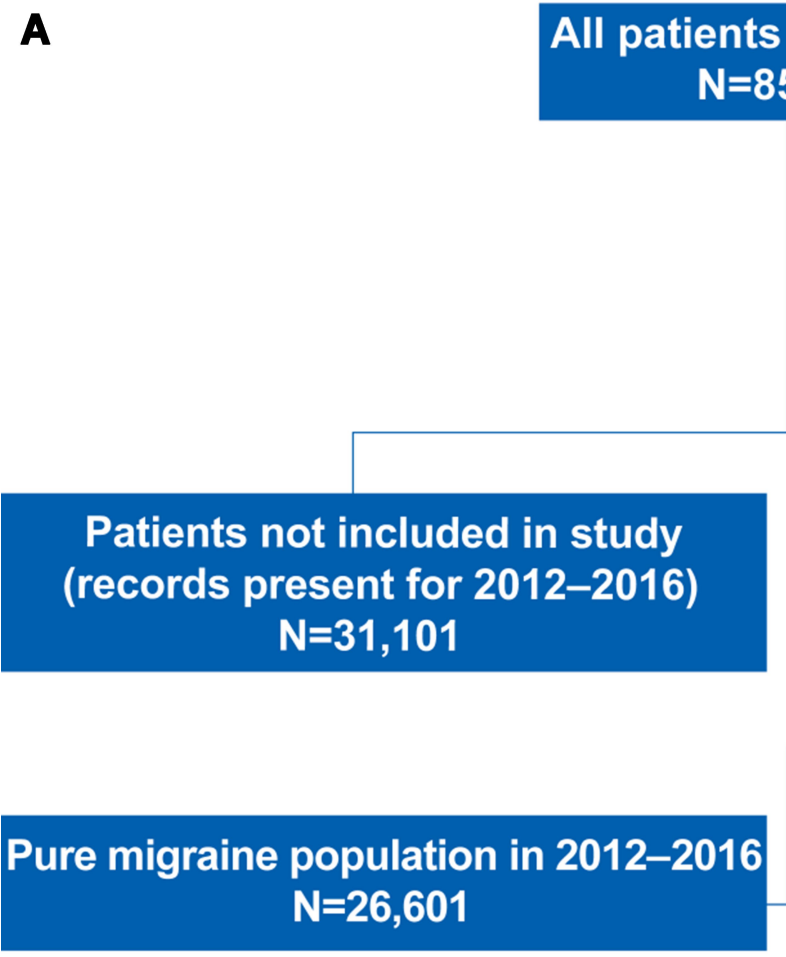

B

Prevalence approach (Index year 2016) Mean age: 42.0 (2-92) years Females: $80.8 \%$

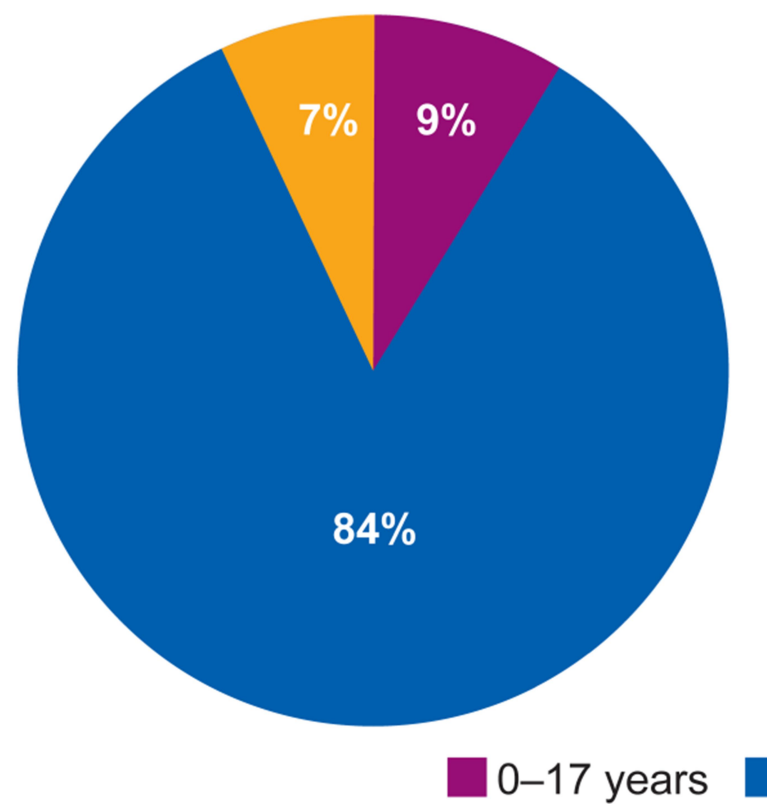

Figure 2 Study Population (A) and Patient Demographics (B and C).

When selectively considering the adults, about $41.7 \%$ (three-year recall period) and 50.4\% (seven-year recall period) of the population were using $\geq 1$ prophylactic medication
Patients not included in study population (records in 2010, 2011, 2017 or 2018) $\mathrm{N}=25,740$

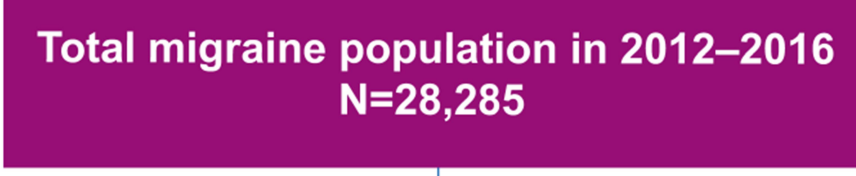

\section{C}

\section{Incidence approach (Index year 2016)}

Mean age: 33.7 (2-92) years

Females: $71.5 \%$

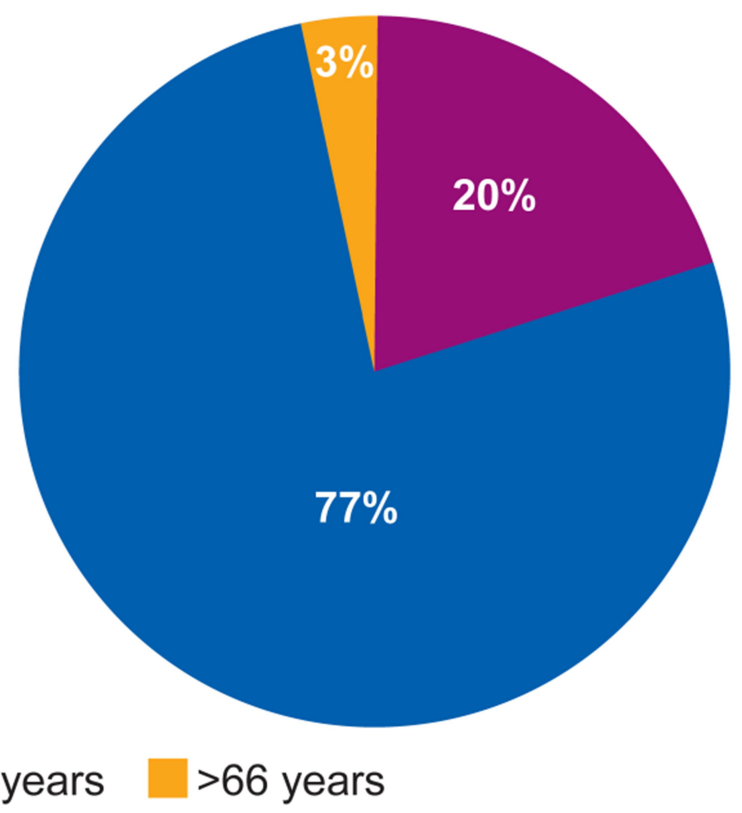

\section{8-65 years $\quad>66$ years}

(Table 2B). At the same time, $12.2 \%$ (three-year recall period) and $21.6 \%$ (seven-year recall period) of the population were observed to be using $\geq 2$ prophylactic medications. 
Table I Migraine Prevalence and Diagnosis Rate in the Population Covered by Government-Paid Healthcare

\begin{tabular}{|l|l|l|l|l|l|l|}
\hline \multirow{2}{*}{$\begin{array}{l}\text { Index } \\
\text { Year }\end{array}$} & \multicolumn{3}{|l|}{ Prevalence Approach } & \multicolumn{2}{l|}{ Incidence Approach } \\
\cline { 2 - 7 } & $\begin{array}{l}\text { Average Size of } \\
\text { Insured Population }\end{array}$ & $\begin{array}{l}\text { Number of } \\
\text { Migraine } \\
\text { Patients }\end{array}$ & $\begin{array}{l}\text { Migraine } \\
\text { Prevalence Rate } \\
(\%)\end{array}$ & $\begin{array}{l}\text { Average Size of } \\
\text { Insured Population }\end{array}$ & $\begin{array}{l}\text { Number of } \\
\text { Migraine } \\
\text { Patients }\end{array}$ & $\begin{array}{l}\text { Migraine } \\
\text { Diagnosis Rate } \\
(\%)\end{array}$ \\
\hline 2012 & $1,186,766$ & 12,233 & 1.03 & $1,186,766$ & 4379 & 0.37 \\
2013 & $1,205,627$ & 12,384 & 1.03 & $1,205,627$ & 3564 & 0.30 \\
2014 & $1,239,890$ & 13,158 & 1.06 & $1,239,890$ & 3623 & 0.29 \\
2015 & $1,260,834$ & 13,528 & 1.07 & $1,260,834$ & 3297 & 0.26 \\
2016 & $1,288,409$ & 14,030 & 1.09 & $1,288,409$ & 3049 & 0.24 \\
\hline
\end{tabular}

Notes: Migraine prevalence/diagnosis rate calculated as the percentage of migraine patients out of the average insured population size covered by the Ministry of the Interior Health Insurance Fund healthcare insurance company. ${ }^{a}$ The diagnosis rate decreases from 2012 to 2016 as for the reference year 2012 , years covered were 2010-201I whereas for the reference year 2016, years covered were 2010-2015.

Among the newly diagnosed patients (patients analyzed by the incidence approach and by the three-year recall period), $86.9 \%$ of the population did not use any prophylactic medication. The mean age of patients ranged between 40 and 48 years of age for the $\geq 1$ to $\geq 4$ prophylactic lines, and $13.1 \%$ and $1.8 \%$ of the total patients were using $\geq 1$ and $\geq 2$ prophylactic medications, respectively. Approximately $84 \%$ of patients using $\geq 2$ prophylactic medications were females (Table $2 \mathrm{C}$ and $\mathrm{D}$ ).

Proportion of Each Therapeutic Subgroup/Medication Used Among the Migraine Patients

The most prescribed therapeutic subgroups were antiepileptics $(26 \%$ with the prevalence approach [antiepileptics other than topiramate: $15.8 \%$, topiramate: $10.1 \%$ ] and $9 \%$ with the incidence approach [antiepileptics other than topiramate: $5.7 \%$, topiramate: $3.2 \%$ ]) and beta blockers (15.8\% for the prevalence approach and $3.5 \%$ for the incidence approach) (Table 3). The most prescribed prophylactic medications using the prevalence approach were topiramate $(10.1 \%)$ and metoprolol $(9.6 \%)$, while newly diagnosed patients mostly used topiramate (3.2\%) and pregabalin $(2.2 \%)$, followed by metoprolol $(2.0 \%)$ (Table 4).

\section{Acute Treatment}

Acute treatment comprised only of triptans, and 4299 patients (30.6\%, N=14,030; prevalence approach) and 788 patients $(25.8 \%, \mathrm{~N}=3049$; incidence approach) who used triptans were not on a prophylactic drug therapy (Figure 3). For assessment of concomitant prophylactics, distinct/exact medication lines $(1,2,3$, and 4) were considered. With the incidence approach, we observed that patients using more than one prophylactic drug chose (or were prescribed) only sumatriptan. In the case of patients using only one prophylactic drug, of the 164 triptan therapy patients (incidence approach), 155 were using only sumatriptan. Similar observations were made using the prevalence approach, wherein 1487 among the 1677 patients on triptans with one prophylactic drug were on sumatriptan.

Overall, sumatriptan was being used by 6021 patients (approximately 43\%; prevalence approach) and 934 patients (30.6\%; incidence approach) (Figure 3). In the prevalence approach, the proportion of patients using triptans decreased with an increase in the number of simultaneous prophylactic drugs used.

\section{Number of Migraine Patients with Comorbidities}

The insurance claims database did not register comorbidities for most migraine patients $(98.6 \%$ by the prevalence approach and $99.2 \%$ by the incidence approach), and the comorbidities recorded were mostly cardiovascular disorders $(1.34 \%$, prevalence approach; 0.91\%, incidence approach).

\section{Number of Patients Who Could Be Eligible for CGRP Therapy}

In the seven-year recall period, about $20 \%$ of patients (all age groups) and $21.6 \%$ of patients (adults only) used $\geq 2$ prophylactic therapies. Considering the number of inhabitants in the Czech Republic (10.7 million), the $1 \%$ migraine prevalence rate (with respect to insured healthcare) and the $21.6 \%$ patients on $\geq 2$ prophylactic medications, there could be up to 23,000 adult patients eligible for CGRP pathway-targeting therapies (Figure 4). 
Table 2 Prophylactic Treatment Patterns for Migraine Patients

\begin{tabular}{|c|c|c|c|c|c|}
\hline \multicolumn{6}{|c|}{ A Descriptive Patient Characteristics (Age and Sex) by Cumulative Prophylactic Line (Prevalence Approach) } \\
\hline \multirow{4}{*}{ Prophylactic Line $^{a}$} & \multicolumn{5}{|l|}{ All Age Groups $(N=14,030)$} \\
\hline & \multicolumn{2}{|l|}{ Age (Years) } & \multicolumn{3}{|l|}{ Females } \\
\hline & 3-Year Recall (2014-2016) & 7-Year Recall (2010-2016) & \multicolumn{2}{|c|}{ 3-Year Recall (2014-2016) } & 7-Year Recall (2010-2016) \\
\hline & Mean (SD) & Mean (SD) & \multicolumn{2}{|l|}{$\%$} & $\%$ \\
\hline 0 & $37.8(15.6)$ & $36.7(15.7)$ & \multicolumn{2}{|l|}{79.1} & 77.8 \\
\hline$\geq 1$ & 48.5 (14.6) & $48.0(14.4)$ & \multicolumn{2}{|l|}{83.5} & 84.2 \\
\hline$\geq 2$ & $50.6(13.2)$ & $50.0(13.4)$ & \multicolumn{2}{|l|}{84.6} & 86.0 \\
\hline$\geq 3$ & $51.7(12.5)$ & $51.6(12.6)$ & \multicolumn{2}{|l|}{84.4} & 86.6 \\
\hline$\geq 4$ & $53.1(12.6)$ & $51.9(11.7)$ & \multicolumn{2}{|l|}{89.0} & 87.6 \\
\hline \multicolumn{6}{|c|}{ B Number of Migraine Patients by Cumulative Prophylactic Line (Prevalence Approach) } \\
\hline \multirow[t]{3}{*}{ Prophylactic Line $^{\mathrm{a}}$} & \multicolumn{2}{|l|}{ All Age Groups $(N=14,030)$} & \multicolumn{3}{|c|}{ Age Group $>18$ Years $(N=\mid 2,780)$} \\
\hline & 3-Year Recall (2014-2016) & 7-Year Recall (2010-2016) & \multicolumn{2}{|c|}{ 3-Year Recall (20I4-20I6) } & 7-Year Recall (2010-2016) \\
\hline & n (\%) & n (\%) & \multicolumn{2}{|l|}{ n (\%) } & n (\%) \\
\hline 0 & $8584(61.2)$ & $7462(53.2)$ & \multirow{5}{*}{\multicolumn{2}{|c|}{$\begin{array}{l}7452(58.3) \\
5336(41.7) \\
1558(12.2) \\
415(3.2) \\
91(0.7)\end{array}$}} & $6348(49.6)$ \\
\hline$\geq 1$ & $5446(38.8)$ & $6568(46.8)$ & & & $6440(50.4)$ \\
\hline$\geq 2$ & $1567(11.2)$ & $2775(19.8)$ & & & $2756(21.6)$ \\
\hline$\geq 3$ & $417(3.0)$ & $1087(7.8)$ & & & $1085(8.5)$ \\
\hline$\geq 4$ & $91(0.6)$ & $370(2.6)$ & & & $370(2.9)$ \\
\hline \multicolumn{6}{|c|}{ C Descriptive Patient Characteristics (Age and Sex) by Cumulative Prophylactic Line; 3-Year Recall Period (2014-2016) (Incidence Approach) } \\
\hline & \multicolumn{5}{|l|}{ All Age Groups ( $N=3049)$} \\
\hline \multirow[t]{2}{*}{ Prophylactic Line ${ }^{a}$} & \multicolumn{3}{|l|}{ Age (Years) } & \multicolumn{2}{|l|}{ Females } \\
\hline & \multicolumn{3}{|l|}{ Mean (SD) } & \multicolumn{2}{|l|}{$\%$} \\
\hline 0 & \multicolumn{3}{|l|}{$32.7(16.1)$} & \multicolumn{2}{|l|}{70.6} \\
\hline$\geq 1$ & $40.1(14.2)$ & & & 77.4 & \\
\hline$\geq 2$ & $43.6(13.9)$ & & & 83.9 & \\
\hline$\geq 3$ & $44.2(12.8)$ & & & 91.7 & \\
\hline$\geq 4$ & $48(10.6)$ & & & 66.7 & \\
\hline D Number of Migra & Patients by Cumulative Pr & lactic Line; 3-Year Recall Pe & d (2014-20IC & (Incidence A & proach) \\
\hline Prophylactic Line ${ }^{a}$ & All Age Groups ( $N=3049)$ & & & & \\
\hline & n & & & $\%$ & \\
\hline 0 & 2651 & & & 86.9 & \\
\hline$\geq 1$ & 398 & & & 13.1 & \\
\hline$\geq 2$ & 56 & & & 1.8 & \\
\hline$\geq 3$ & 12 & & & 0.4 & \\
\hline$\geq 4$ & 3 & & & 0.1 & \\
\hline
\end{tabular}

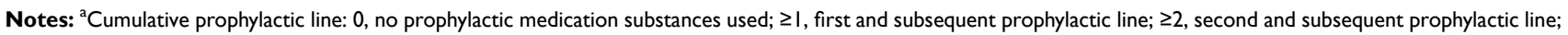
$\geq 3$, third and subsequent prophylactic line; $\geq 4$, fourth and subsequent prophylactic line.

Abbreviations: $n$, number of migraine patients by cumulative prophylactic line; $N$, total number of migraine patients; \%, percentage of migraine patients with cumulative prophylactic lines out of the overall number of migraine patients with respect to the analysis approach and by the analysis population; SD, standard deviation.

\section{Discussion}

There have been various studies across Europe and in other continents comparing the clinical and economic burden of migraine using various strategies as aforementioned. ${ }^{12,14,15}$ The Burden of Episodic and Chronic Migraine in Europe (BECOME) study evaluated the burden of migraine across 
Table 3 Proportion of Migraine Patients Using Prophylactic Medication by Therapeutic Subgroup; 3-Year Recall Period

\begin{tabular}{|l|l|l|l|l|}
\hline \multirow{2}{*}{ Therapeutic Subgroup } & \multicolumn{2}{|l|}{$\begin{array}{l}\text { Prevalence } \\
\text { Approach } \\
\text { (N=14,030) }\end{array}$} & \multicolumn{2}{|l|}{$\begin{array}{l}\text { Ancidence } \\
\text { Approach } \\
(\mathbf{N = 3 0 4 9 )}\end{array}$} \\
\cline { 2 - 5 } & $\mathbf{n}$ & $\%$ & $\mathbf{n}$ & $\%$ \\
\hline Antiepileptics Other Than Topiramate & 2222 & 15.8 & 173 & 5.7 \\
Beta Blockers & 2213 & 15.8 & 106 & 3.5 \\
Botulinum Toxin A & 49 & 0.4 & 4 & 0.1 \\
Selected Antidepressants & 1015 & 7.2 & 66 & 2.2 \\
Topiramate & 1421 & 10.1 & 96 & 3.2 \\
\hline
\end{tabular}

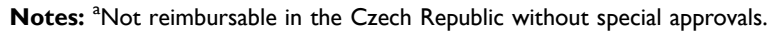
Abbreviations: $N$, total number of migraine patients in 2016; n, number of migraine patients in 2016 by therapeutic subgroup; \%, percentage of migraine patients in the therapeutic subgroup out of the overall number of migraine patients in 2016 with respect to the analysis approach and by the analysis population.

Table 4 Proportion of Migraine Patients Using Prophylactic Medication; Individual Drugs, 3-Year Recall Period

\begin{tabular}{|l|l|l|l|l|}
\hline \multirow{2}{*}{ Substance } & \multicolumn{2}{l|}{$\begin{array}{l}\text { Prevalence Approach } \\
\text { (N=14,030) }\end{array}$} & \multicolumn{2}{l|}{$\begin{array}{l}\text { Incidence Approach } \\
\text { (N=3049) }\end{array}$} \\
\cline { 2 - 5 } & $\mathbf{n}$ & $\%$ & $\mathbf{n}$ & $\%$ \\
\hline No Medication & 8584 & 61.2 & 2651 & 86.9 \\
Metoprolol & 1342 & 9.6 & 60 & 2.0 \\
Atenolol & 138 & 0.9 & 5 & 0.2 \\
Bisoprolol & 868 & 6.2 & 44 & 1.4 \\
Candesartan & 115 & 0.8 & 6 & 0.2 \\
Botulinum Toxin A & 49 & 0.4 & 4 & 0.1 \\
Valproic Acid & 826 & 5.9 & 58 & 1.9 \\
Topiramate & 1421 & 10.1 & 96 & 3.2 \\
Gabapentin & 939 & 6.7 & 61 & 2.0 \\
Pregabalin & 791 & 5.6 & 68 & 2.2 \\
Amitriptyline & 341 & 2.4 & 28 & 0.9 \\
Venlafaxine & 722 & 5.2 & 40 & 1.3 \\
\hline
\end{tabular}

Abbreviations: $n$, number of migraine patients with the corresponding prophylactic medication prescribed in 2016; N, total number of migraine patients in 2016; \%, percentage of migraine patients with the corresponding prophylactic medication out of the overall number of migraine patients in 2016 with respect to the analysis approach and by the analysis population.

17 European countries (including the Czech Republic) and Israel, but involved only tertiary healthcare centers. ${ }^{20}$ In our study, the year 2016 was the last index year. However, based on the methodology applied, data from the years 2017 and 2018 were also used for the analyses. When data request was made to the database owner (ZPMV; in 2019), the data from the year 2018 was the latest available data for that complete year. Thus, the data presented here have been compiled using the latest information.

Our study included data on primary and secondary healthcare on a national level, thus providing a broader overview of migraine management in the country. Most of the migraine patients were adults in their productive years and females, as observed in other studies. ${ }^{21-24}$ Migraine prevalence rate remained stable during 2012-2016 and migraine diagnosis rate was $0.2-0.4 \%$. Antiepileptics and beta blockers were observed to be the most commonly used prophylactic drugs. By prevalence approach, antiepileptics (topiramate) and beta blockers (metoprolol) were the most prescribed drugs, and the antiepileptics topiramate and pregabalin were mostly used by newly diagnosed patients. There is similar evidence supporting the use of beta blockers such as metoprolol, ${ }^{7,25}$ and antiepileptic drugs such as topiramate as preventive medication for migraine. ${ }^{7,26}$

Worldwide, treatment patterns vary because of reimbursement guidelines and requirements and differences in the prescribing patterns of healthcare practitioners. We observed that $13-39 \%$ of patients (depending upon prevalence or incidence approach) were on prophylactic medication with the three-year recall period, and when taking into account the seven-year recall period by the prevalence approach, the proportion of patients on prophylactic medication was about 47\% (all age groups) and 50\% (adults only). This clearly denotes that prophylactic therapy in the Czech Republic is underutilized. Additionally, in view of the European ${ }^{27}$ and United States ${ }^{4}$ recommendations for use of CGRP pathway-targeting therapies in patients with two prior prophylactic treatment failures, we deduced that 23,000 adult patients could be eligible for CGRP therapies.

\section{Strengths and Limitations}

Only $0.2-1 \%$ of the total insured population had migrainerelated expenses towards general or government-paid healthcare. This maybe because majority of the migraine sufferers experienced mild migraine and used over-thecounter drugs such as general analgesics. Analgesics are mostly used against acute migraine and are not claimed because they cannot be reimbursed. This maybe also be a possible reason for our inability to capture acute treatment forms other than triptan therapy in the Czech Republic. Among the triptans, sumatriptan appeared to be the most trusted medication for acute treatment. Of note was the observation that $25-30 \%$ of the patients did not use any prophylactic drug along with triptan therapy (in both the prevalence and incidence approaches), and that triptan usage decreased in patients as the number of prophylactic drugs in use increased (prevalence approach). The latter observation agrees with one of the objectives of 


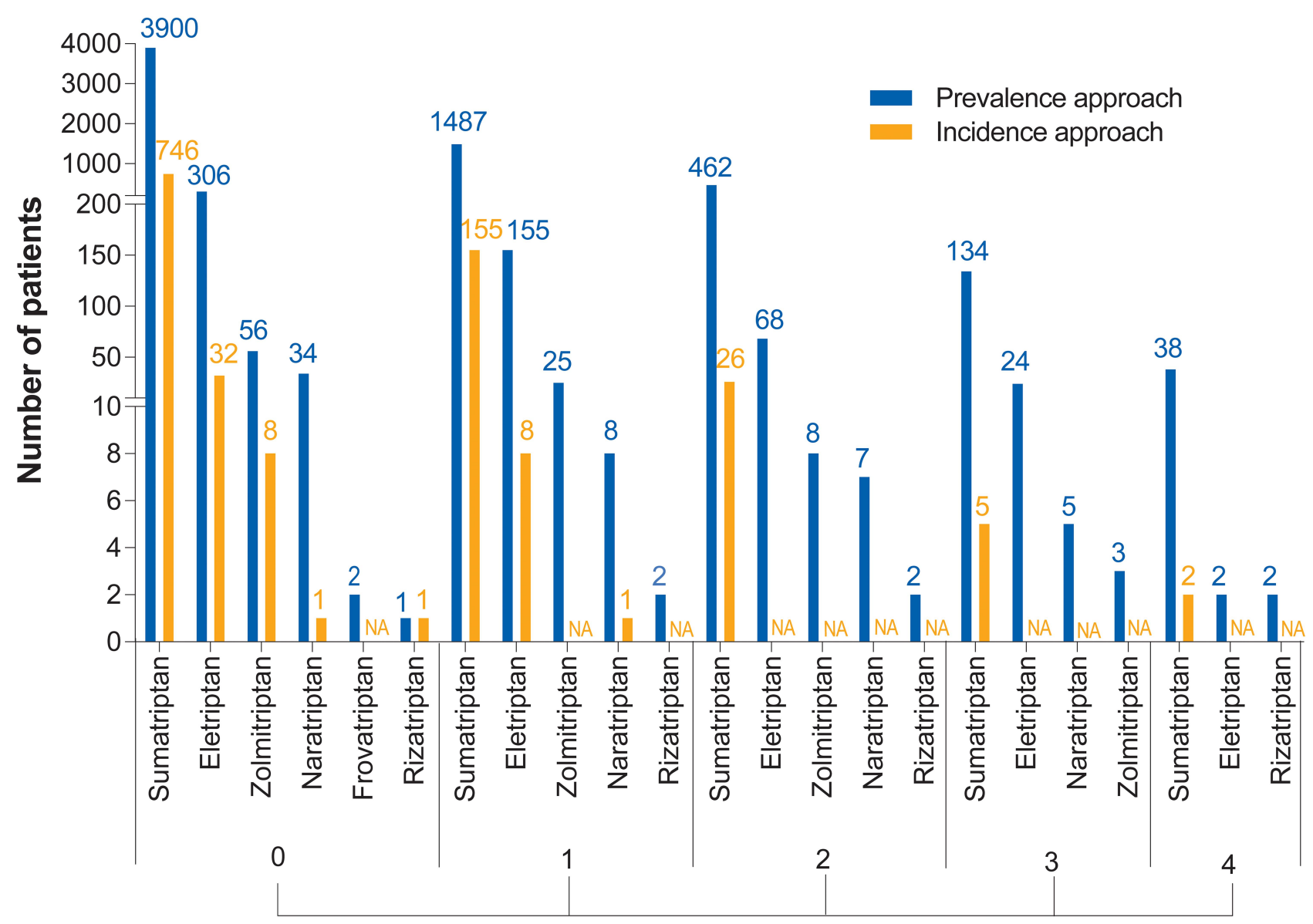

Concomitant prophylactic medications

Figure 3 Number of Migraine Patients Using Triptans Acutely While on Prophylactic Therapy in 2016. Abbreviation: NA, not applicable.

prophylactic treatment (to reduce the frequency of acute medication) and is in harmony with other studies. ${ }^{6,7,28}$ Comorbidities were not well captured (only $1-2 \%$ of patients had recorded comorbidities) in this study since claims data does not describe every medical facet as customarily only the primary diagnosis is claimed. Moreover, if care is provided by general practitioners who are paid by capitation, the patients do not make health insurance claims since they will not be reimbursed. One unavoidable limitation in the study was the possible misidentification of newly diagnosed patients under the incidence approach. Many migraine patients do not feel the need to visit a physician or undergo any therapy until their symptoms aggravate. Consequently, relapsing patients (diagnosed before the index year) may have been included in the newly diagnosed patient population. Data obtained through the seven-year recall period provided a clear depiction of the number of long-term migraine sufferers, including those with prior treatment failures with more than two prophylactic drugs. This helps us understand the number of patients who are in need of CGRP therapy in the Czech Republic.

\section{Conclusion}

Based on the health insurance claimed data, one-third of the total migraine population in the Czech Republic comprised newly diagnosed patients, and the prevalence rate of migraine remained stable during 2012-2016. The country's treatment patterns were similar to the worldwide trend; migraine has been managed with antiepileptics and beta blockers in the preventive setting and with triptans for acute therapy. Utilization of prophylactic therapy was associated with a decreased need for acute management of migraine as demonstrated by triptan use, which was higher in patients who were not on prophylactic treatment or were on a single prophylactic. There was a reduction in triptan use with combination prophylactics. Our study shows that there is room for improvement in the use of conventional 


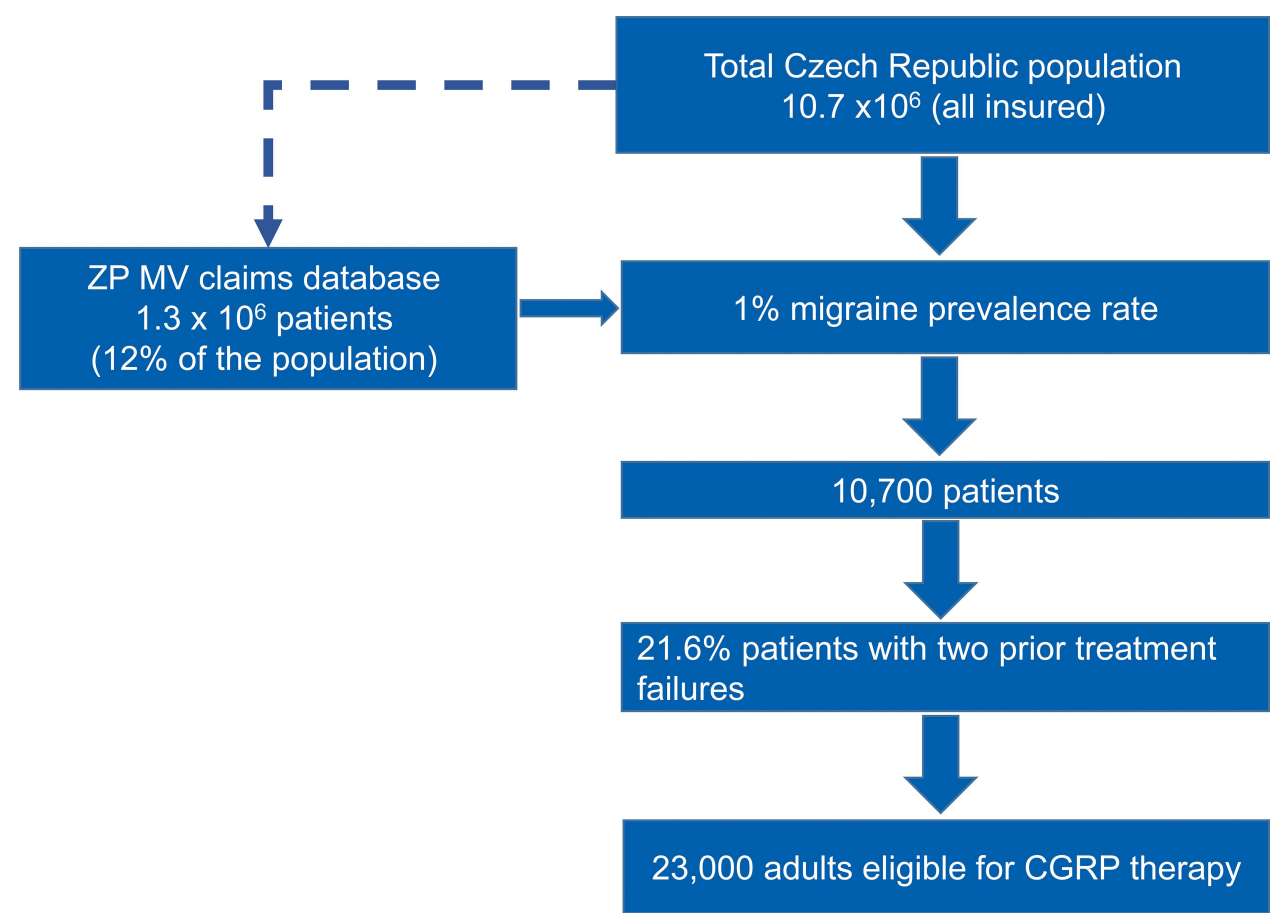

Figure 4 Number of Migraine Patients Who Could Be Eligible for CGRP Therapy in the Czech Republic. Abbreviation: CGRP, calcitonin gene-related peptide.

prophylactic treatment, as we still see the use of anticonvulsants - albeit with a lower level of evidence (eg, gabapentin, pregabalin) - for migraine prophylaxis. About $50 \%$ of adult migraine patients were on preventive therapy and an estimated 23,000 individuals in the Czech Republic could be eligible for recent therapies using CGRP pathway antagonists such as erenumab. The methodology used in our study can be easily transferred/applied to other data/databases in other countries, and it might be a valid source of epidemiological data (also in terms of proportion of patients eligible for CGRP-mediated therapies) for other countries. This is because these conventional prophylactic treatment patterns might not vary substantially across the world, particularly in the European region. It will be worthwhile to observe the effects of CGRP therapies on a national level in the Czech Republic in order to obtain a broader and more comprehensive understanding of its effectiveness as well as adoption as a standard in migraine management.

\section{Acknowledgments}

The authors thank Shyamashree Dasgupta (Medical Communications, Novartis Healthcare Pvt. Ltd) for assistance in writing the manuscript, formatting, referencing, preparing tables and figures, incorporating the authors' revisions, and submission, all under the direction of the authors.
The final responsibility for the content lies with the authors. The authors thank ZPMV for providing the data for the analysis. The study was funded by Novartis, s.r.o. Erenumab is co-developed by Novartis and Amgen, and Novartis provided medical writing support for the preparation of this manuscript. No ethics approval was required for this study as it involved no human/animal subjects. Data obtained was from a database. Permission was obtained from the database owner (ZPMV) to use the data in the study. Part of the results mentioned in this manuscript were presented via poster on 19th Congress of the International Headache Society, Dublin, Ireland, 5-8 September, 2019.

\section{Author Contributions}

David Dolezil, Jolana Markova, Jiri Klimes, Zuzana Pocikova, Filip Dostal and Adam Svobodnik conceived and supervised the study. David Dolezil and Jiri Klimes were involved in developing the methodology, validation and investigation, and Jiri Klimes has full access to the data in the study. Jiri Klimes, Zuzana Pocikova and Filip Dostal were involved in visualization; Jiri Klimes and Zuzana Pocikova also played roles in project administration, funding acquisition and providing resources. Radka Stepanova and Adam Svobodnik performed all formal analyses during the study. All authors contributed to data analysis, drafting or revising 
the article, have agreed on the journal to which the article will be submitted, gave final approval of the version to be published, and agree to be accountable for all aspects of the work.

\section{Disclosure}

David Dolezil has received consulting fees, speaking and/ or teaching fees from Allergan, Amgen, Biogen Idec, Eli Lilly, Novartis, Bayer, and Teva, and has not received any funding related to the development of this manuscript. Jolana Markova has received consulting fees, speaking and/or teaching fees from Almirall, Amgen, Novartis and Teva, and study support from PROGRES Q35/LF3. Jiri Klimes, Filip Dostal and Zuzana Pocikova are Novartis employees. Radka Stepanova and Adam Svobodnik were funded by Novartis for the statistical analyses. The authors report no other conflicts of interest in this work.

\section{References}

1. GBD Neurology Collaborators. Global, regional, and national burden of neurological disorders, 1990-2016: a systematic analysis for the Global Burden of Disease Study 2016. Lancet Neurol. 2019;18(5):459-480.

2. Stovner LJ, Nichols E, Steiner TJ; GBD Headache Collaborators. Global, regional, and national burden of migraine and tension-type headache, 1990-2016: a systematic analysis for the Global Burden of Disease Study 2016. Lancet Neurol. 2018;17(11):954-976. doi:10.1016/S1474-4422(18)30322-3

3. Reuter U. GBD 2016: still no improvement in the burden of migraine. Lancet Neurol. 2018;17(11):929-930. doi:10.1016/S14744422(18)30360-0

4. American Headache Society. The American headache society position statement on integrating new migraine treatments into clinical practice. Headache. 2019;59(1):1-18.

5. Vargas BB. Acute treatment of migraine. Continuum (Minneap Minn). 2018;24(4, Headache):1032-1051.

6. Schwedt TJ. Preventive therapy of migraine. Continuum (Minneap Minn). 2018;24(4, Headache):1052-1065.

7. Silberstein SD. Preventive migraine treatment. Continuum (Minneap Minn). 2015;21(4Headache):973-989.

8. Edvinsson L. Role of CGRP in Migraine. Handb Exp Pharmacol. 2019;255:121-130.

9. Lattanzi S, Brigo F, Trinka E, et al. Erenumab for preventive treatment of migraine: a systematic review and meta-analysis of efficacy and safety. Drugs. 2019;79(4):417-431. doi:10.1007/s40265-019-01069-1

10. Han L, Liu Y, Xiong H, Hong P. CGRP monoclonal antibody for preventive treatment of chronic migraine: an update of meta-analysis. Brain Behav. 2019;9(2):e01215. doi:10.1002/brb3.1215

11. Wang SJ, Chen PK, Fuh JL. Comorbidities of migraine. Front Neurol. 2010;1:16.
12. Bloudek LM, Stokes M, Buse DC, et al. Cost of healthcare for patients with migraine in five European countries: results from the International Burden of Migraine Study (IBMS). $J$ Headache Pain. 2012;13(5):361-378.

13. Linde M, Gustavsson A, Stovner LJ, et al. The cost of headache disorders in Europe: the Eurolight project. Eur J Neurol. 2012;19 (5):703-711

14. Martelletti P, Schwedt TJ, Lanteri-Minet M, et al. My migraine voice survey: a global study of disease burden among individuals with migraine for whom preventive treatments have failed. $J$ Headache Pain. 2018;19(1):115. doi:10.1186/s10194-018-0946-Z

15. Vo P, Paris N, Bilitou A, et al. Burden of migraine in Europe using self-reported digital diary data from the migraine Buddy(c) application. Neurol Ther. 2018;7(2):321-332.

16. Wu C-S, Lai M-S, Gau SS-F, Wang S-C, Tsai H-J. Concordance between patient self-reports and claims data on clinical diagnoses, medication use, and health system utilization in Taiwan. PLoS One. 2014;9(12):e112257. doi:10.1371/journal.pone.0112257

17. Neubauer S, Kreis K, Klora M, Zeidler J. Access, use, and challenges of claims data analyses in Germany. Eur J Health Econ. 2017;18 (5):533-536. doi:10.1007/s10198-016-0849-3

18. Pavlovic JM, Yu JS, Silberstein SD, et al. Development of a claims-based algorithm to identify potentially undiagnosed chronic migraine patients. Cephalalgia. 2019;39(4):465-476.

19. Czech Statistical Office. Public database CZSO 2019. Available from: https://www.czso.cz/csu/czso/population. Accessed December 10, 2019.

20. Lucas C, Pozo Rosich P, Watson D, et al. A real-world analysis of the burden of migraine in patients with prior treatment failures: evidence from the become study. J Headache Pain. 2019;20(1):109.

21. Borsook D, Dodick DW. Taking the headache out of migraine. Neurol Clin Pract. 2015;5(4):317-325. doi:10.1212/CPJ.0000000000000171

22. Sokolovic E, Riederer F, Szucs T, Agosti R, Sándor PS. Self-reported headache among the employees of a Swiss university hospital: prevalence, disability, current treatment, and economic impact. J Headache Pain. 2013;14(1):29. doi:10.1186/1129-2377-14-29

23. World Health Organization. Headache disorders 2016. World Health Organization. Available from: https://www.who.int/news-room/factsheets/detail/headache-disorders. Accessed December 30, 2019.

24. Vetvik KG, MacGregor EA. Sex differences in the epidemiology, clinical features, and pathophysiology of migraine. Lancet Neurol. 2017;16(1):76-87.

25. Jackson JL, Kuriyama A, Kuwatsuka Y, et al. Beta-blockers for the prevention of headache in adults, a systematic review and meta-analysis. PLoS One. 2019;14(3):e0212785.

26. Linde M, Mulleners WM, Chronicle EP, McCrory DC. Topiramate for the prophylaxis of episodic migraine in adults. Cochrane Database Syst Rev. 2013;6:Cd010610.

27. Sacco S, Bendtsen L, Ashina M, et al. European headache federation guideline on the use of monoclonal antibodies acting on the calcitonin gene related peptide or its receptor for migraine prevention. J Headache Pain. 2019;20(1):6. doi:10.1186/s10194-018-0955-y

28. Smelt AF, Assendelft WJ, van Dijk CE, Blom JW. Triptan use after starting prophylactic migraine treatment: a retrospective cohort study in a primary care population. Cephalalgia. 2014;34(11):927-932. doi: $10.1177 / 0333102414521511$ 


\section{Publish your work in this journal}

The Journal of Pain Research is an international, peer reviewed, open access, online journal that welcomes laboratory and clinical findings in the fields of pain research and the prevention and management of pain. Original research, reviews, symposium reports, hypothesis formation and commentaries are all considered for publication. The manuscript management system is completely online and includes a very quick and fair peer-review system, which is all easy to use. Visit http:// www.dovepress.com/testimonials.php to read real quotes from published authors.

Submit your manuscript here: https://www.dovepress.com/journal-of-pain-research-journal 